\title{
Validation of an Optical Flow Method for Tag Displacement Estimation
}

\author{
Lawrence Dougherty Ph.D. ${ }^{1}$, Jane C. Asmuth M.S.E.E. ${ }^{2}$, Aaron Blom B.A. ${ }^{1}$, Rakesh Kumar Ph.D. ${ }^{2}$, Leon Axel M.D., Ph.D. ${ }^{1}$ \\ ${ }^{1}$ Department of Radiology, Hospital of the University of Pennsylvania, Philadelphia, PA \\ ${ }^{2}$ Sarnoff Corporation, Princeton, NJ
}

Introduction We present an optical flow method for the rapid estimation of deformation of magnetic resonance tagged cardiac images. The registration and change visualization (RCV) software is a hierarchical estimation technique that is based on the methods developed by Bergen, et al [1], and adapted for medical applications by Kumar, et al [2]. This method does not rely on prior knowledge of image content and overcomes the requirement of constant pixel intensity in standard optical flow methods by pre-processing the input images to eliminate any intensity bias which results from the reduction in stripe contrast throughout the cardiac cycle.

The most common method presently used to identify tag intersections is a semi-automatic technique based on the active contours model or "snakes" [3]. Although this method is a great improvement over manually tracking the stripe intersections, the stripe fitting must still be monitored and edited in many cases. Identification of the stripe intersections takes approximately 5-10 minutes per slice per cardiac phase. In comparison to the snakes estimation method, the $\mathrm{RCV}$ method is not only significantly faster ( $<5 \mathrm{sec} / \mathrm{slice} / \mathrm{phase})$, but can also track every pixel in the image.

To validate this method in estimating local myocardial deformation, a multi-phase SPAMM [4] tagged series of images of a canine heart was acquired, and the pixel displacement as estimated by the RCV software was compared to that obtained with the snakes method.

Methods Tagged canine heart images were acquired using a whole-body MR scanner (GE Medical Systems, Milwaukee, WI) that uses high speed magnetic field gradient sets $(1.447$ $\mathrm{T} / \mathrm{cm} / \mathrm{s}$ slew rate). A $6 \mathrm{~mm}$ short axis slice in the mid-ventricle was acquired with a fast gradient echo sequence (FGRE) utilizing segmented k-space acquisition. Cardiac tags were applied using the SPAMM sequence synchronous with detection of the QRS complex, and 12 phases within the RR interval were acquired over 32 heart beats. Scan parameters were: $\mathrm{TR}=8.8 \mathrm{~ms}, \mathrm{TE}=2.2 \mathrm{~ms}, 4$ views $/ \mathrm{k}$-space segment, $256 \mathrm{x}$ 128 raw data matrix, $16 \mathrm{~cm}$ FOV $15^{\circ}$ excitation flip angle, and $3 \mathrm{~mm}$ inter-stripe spacing.

For the RCV analysis, the images were sequentially pair-wise compared to compute the flow field that described warping the image of one cardiac phase into alignment with the next. The registration is performed in a multistep procedure that first estimates a global parametric transformation (rigid translation and rotation) between two images, and then, using the global transformation as an initialization, estimates the local flow vector for each point between the two images. The alignment process is initiated by filtering the images using a Laplacian filter, which eliminates any local offset field and enhances the edges in the image. The effect of this filtering is to make the alignment process sensitive to structure and not overall intensity. The motion parameters, $\mathbf{u}(\mathbf{x})$, are computed for each adjacent pair of images which minimize the sum of the square differences (SSD) between the two images. These parameters can either be computed globally or locally. The minimization of the SSD with respect to $\mathbf{u}$ is an iterative process. For each iteration, we compute the incremental change in velocity using the Gauss-Newton optimization technique, which uses a first order expansion of the individual error quantities before squaring. The incremental estimate $\partial \mathbf{u}$ is computed and added to the current estimate $\mathbf{u}_{\mathrm{i}}$ to obtain a new estimate $\mathbf{u}_{i+1}$. This process is repeated for a fixed number of iterations or until there is convergence

The snakes method was used to identify the stripe intersections in the end-diastolic and end-systolic images, from which the end-systolic vector displacement was calculated for each estimated intersection. Triangular regions of interest were constructed from the intersections, and the displacement at the centroid of the triangle calculated by taking the average of the displacements of the vertices

Results A multi-phase short axis slice of a canine heart was acquired using the SPAMM tagged FGRE sequence. From the snakes analysis, 172 points were identified as stripe intersections in the myocardium of the left ventricle and 222 triangles were constructed from these points. The vector displacement to end-systole of each point was determined and the displacement value for the centroid of the triangles was calculated. Using the RCV method of displacement estimation, 22800 points were sequentially tracked to end-systole, of which $\sim 6000$ points were from the myocardium. The pixel displacements from each phase were vector integrated and masked [Figure 1]. These data were decimated by taking the median value within a $5 \times 5$ window at the centroid of each triangle used in the snake method. The RMS error between the RCV method and the snake method is 0.29 pixels.

Conclusion The RCV method of estimating MR tag displacements has proven to be as accurate as the snakes method. From the canine study, the RMS error in computed overall end-systolic displacement of 0.29 pixels indicates accuracy within the measurement error of the snakes method (0.38 pixels [3]). The RCV method has the advantage of tracking all pixels in the image, and in a substantially shorter period than the snakes method. Analysis of a multi-phase multi-slice set of slices covering the left ventricle will take $\sim 10$ min, as opposed to 2 hours using snakes.

\section{References}

1. J.R. Bergen, P. Anadan, K.J. Hanna, R. Hingorani, "Hierarchical model-based motion estimation," Proc. European Conference on Computer Vision, G. Sandini, Ed., Springer-Verlag, 1992.

2. R. Kumar, K. Hanna, J.C. Asmuth, et al, "Detecting lesions in magnetic resonance breast scans," 24th AIPR Workshop, Oct., 1995.

3. D. Kraitchman, A.A. Young, L. Axel , "Semi-automatic tracking of myocardial motion in MR tagged images," IEEE Trans. Med. Imaging, vol. 14, pp. 422-433, 1995.

4. L. Axel, L. Dougherty, "Heart wall motion: Improved method of spatial modulation of magnetization for MR imaging," Radiology, vol. 172, pp. 349-350, 1989.

Figure 1) Short axis image of a canine heart showing end-systolic displacement displayed as intensity on the end-diastolic image, as computed with the RCV method. Image intensity scale is $0-8$ pixels.



